PREPARED FOR THE U.S. DEPARTMENT OF ENERGY, UNDER CONTRACT DE-AC02-76CH03073

PPPL-3826

PPPL-3826

UC-70

Improved Conservation Properties

for Particle-in-cell Simulations with Kinetic Electrons

by

J.L.V. Lewandowski

June 2003

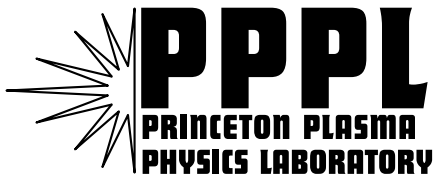

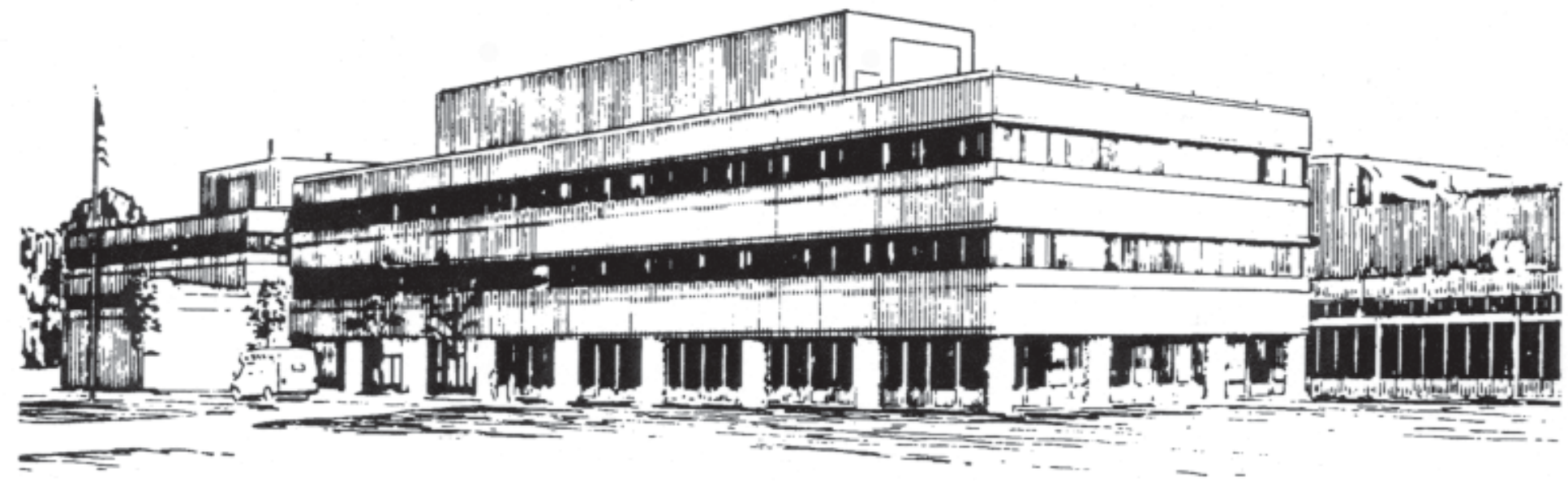

PRINCETON PLASMA PHYSICS LABORATORY PRINCETON UNIVERSITY, PRINCETON, NEW JERSEY 


\section{PPPL Reports Disclaimer}

This report was prepared as an account of work sponsored by an agency of the United States Government. Neither the United States Government nor any agency thereof, nor any of their employees, makes any warranty, express or implied, or assumes any legal liability or responsibility for the accuracy, completeness, or usefulness of any information, apparatus, product, or process disclosed, or represents that its use would not infringe privately owned rights. Reference herein to any specific commercial product, process, or service by trade name, trademark, manufacturer, or otherwise, does not necessarily constitute or imply its endorsement, recommendation, or favoring by the United States Government or any agency thereof. The views and opinions of authors expressed herein do not necessarily state or reflect those of the United States Government or any agency thereof.

\section{Availability}

This report is posted on the U.S. Department of Energy's Princeton Plasma Physics Laboratory Publications and Reports web site in Fiscal Year 2003. The home page for PPPL Reports and Publications is: http://www.pppl.gov/pub_report/

DOE and DOE Contractors can obtain copies of this report from:

U.S. Department of Energy

Office of Scientific and Technical Information

DOE Technical Information Services (DTIS)

P.O. Box 62

Oak Ridge, TN 37831

Telephone: (865) 576-8401

Fax: (865) 576-5728

Email: reports@adonis.osti.gov

This report is available to the general public from:

National Technical Information Service

U.S. Department of Commerce

5285 Port Royal Road

Springfield, VA 22161

Telephone: $1-800-553-6847$ or

(703) $605-6000$

Fax: (703) 321-8547

Internet: http://www.ntis.gov/ordering.htm 


\title{
Improved Conservation Properties for Particle-in-cell Simulations with Kinetic Electrons
}

\author{
J.L.V. Lewandowski * \\ Princeton Plasma Physics Laboratory \\ Princeton University, P.O. Box 451 \\ Princeton NJ 08543 \\ USA
}

June 9, 2003

\begin{abstract}
It is shown that a simple algorithm which exactly segregates between adiabatic and nonadiabatic electrons in particle-in-cell simulations of drift modes yields excellent conservation properties (e.g. particle number, energy) compared to the conventional $\delta f$ scheme. The removal of the free streaming term in the evolution of the marker weight is shown to be responsible for the improved linear and nonlinear properties of the simulated plasma.
\end{abstract}

Pacs \# : 52.35Kt, 52.30Jb, 52.35Ra

\section{Introduction}

It is generally accepted that low-frequency, small-scale instabilities are major contenders for the anomalous transport observed in tokamaks [1-3] and stellarators [4]. Particle-in-cell simulations of ion dynamics in tokamak plasmas is now well understood; however, the numerical study of electron dynamics, including kinetic effects, has received much less attention.

The treatment of electrons in particle-in-cell simulations is made difficult due to the fact that the electrons move $\sim \sqrt{m_{i} / m_{e}} \gg 1$ times faster than the ions. Although the standard

*e-mail: jlewando@pppl.gov 
$\delta f$ scheme [5] works well for the ion dynamics, an accuracy problem arises when the scheme is used to treat the electron dynamics. Therefore, noting that the bulk of the electrons respond adiabatically to the low-frequency $\left(\omega \ll \omega_{c i}\right)$ waves, it may be (physically and numerically) advantageous to focus on the nonadiabatic part of the electron response.

In this Letter, it is shown that an exact segregation between adiabatic and nonadiabatic electron responses yields a more accurate linear response of the plasma (e.g. linear growth rate) and improved nonlinear properties (particle number and energy conservation). The splitting scheme introduced in this Letter can be adapted to study the impact of electron dynamics on ion-temperature-gradient driven modes in general toroidal geometry.

\section{Kinetic Electron Scheme}

In this section, we present a scheme that separates adiabatic and nonadiabatic electron responses for the case of electrostatic drift waves in shearless slab geometry. The collisionless, electrostatic, gyrokinetic Vlasov equation for species $j$ with mass $m_{j}$ and charge $q_{j}$ is, in the long-wavelength limit, given by [6]

$$
\frac{d F_{j}}{d t} \equiv \frac{\partial F_{j}}{\partial t}+\left(v_{\|} \widehat{\mathbf{b}}_{0}+\mathbf{V}_{E}\right) \cdot \nabla F_{j}-\frac{q_{j}}{m_{j}} \widehat{\mathbf{b}}_{0} \cdot \nabla \Phi \frac{\partial F_{j}}{\partial v_{\|}}=0
$$

where $\widehat{\mathbf{b}}_{0}=\mathbf{B}_{0} / B_{0}$ is a unit vector and $\mathbf{V}_{E}=c \widehat{\mathbf{b}}_{0} \times \nabla \Phi / B_{0}$ is the $\mathbf{E} \times \mathbf{B}$ drift velocity. The confining magnetic field is taken to be of the form $\mathbf{B}_{0}=B_{0}(\widehat{\mathbf{z}}+\theta \widehat{\mathbf{y}})$ where $\theta$ is a small parameter. For the standard $\delta f$ scheme, the distribution is written as

$$
F_{j}=F_{M j}+\delta f_{j} ;
$$

where $F_{M j}$ is the Maxwellian distribution. The evolution equation for the marker weight, $W_{j} \equiv$ $\delta f_{j} / F_{j}$, is obtained from Eqs. $(1,2)$ as

$$
\frac{d W_{j}}{d t}=\left(1-W_{j}\right)\left[\left(\widehat{\mathbf{b}}_{0} \times \nabla \Phi\right) \cdot \boldsymbol{\kappa}_{j}-\sigma_{j} v_{\|} \nabla_{\|} \Phi\right]
$$

where gyrokinetic units $\left(\omega_{c i} t \mapsto t ; v_{\|} / c_{s} \mapsto v_{\|} ; \rho_{s} \boldsymbol{\kappa} \mapsto \boldsymbol{\kappa} ; \rho_{s} \boldsymbol{\nabla} \mapsto \boldsymbol{\nabla} ; e \Phi / T_{e} \mapsto \Phi\right)$ are used, together with the definitions of $\boldsymbol{\kappa}_{j}=-\nabla F_{M j} / F_{M j}, \sigma_{j}=Z_{j} T_{e} / T_{j}, \rho_{s}=c_{s} / \omega_{c i}, \omega_{c i}=e B /\left(m_{i} c\right)$ and $c_{s}=\sqrt{T_{e} / m_{i}}$. The electrostatic potential is obtained from the gyrokinetic Poisson equation [6] (in the long-wavelength limit)

$$
\nabla_{\perp}^{2} \Phi=-\rho=\int_{-\infty}^{+\infty}\left(\delta f_{e}-\delta f_{i}\right) d v_{\|}
$$

As discussed in the Introduction, the bulk of the electrons respond adiabatically to low-frequency, drift-type mode $\left(V_{t h e} \gg \omega / k_{\|}\right)$. Therefore, for most of the electrons, the last term on the righthand side of Eq.(3) does not carry much relevant information; however, this term may generate noise, especially in the fully turbulent state. Therefore, in order to remedy this situation, it is natural to separate the electrons into two groups (adiabatic and nonadiabatic) so as to reflect their different responses to the low-frequency waves by writing the distribution $F_{j}$ as

$$
F_{j}=\exp \left(-\frac{q_{j}}{T_{j}} \Phi\right) F_{M j}+h_{j}
$$


The equation governing the weight associated with the nonadiabatic part of the distribution function, $\bar{W}_{j} \equiv h_{j} / F_{j}$, can be easily derived using Eqs.(1,5)

$$
\frac{d \bar{W}_{j}}{d t}=\left(1-\bar{W}_{j}\right)\left(\mathbf{V}_{E} \cdot \boldsymbol{\kappa}_{j}+\frac{q_{j}}{T_{j}} \varphi\right)
$$

where $\varphi \equiv \partial \Phi / \partial t$ is termed the polarization field. By construction, the contribution due to the free streaming markers has been removed from the weight equation [compare with Eq.(3)]. Representation (5) implies the computation of an additional scalar field; a direct discretization of $\varphi=\partial \Phi / \partial t$ in the right-hand side of Eq.(6) can be shown to be numerically unstable. It is preferable to exploit the information contained in the original Vlasov equation, Eq.(1, and the gyrokinetic Poisson equation, Eq.(4). We start by integrating the Vlasov equation over velocity space with the result of

$$
\frac{\partial \rho}{\partial t}=-\mathbf{V}_{E} \cdot \nabla \rho-\nabla_{\|} J_{\|}
$$

where $J_{\|}$is the parallel current density. Taking the time derivative of Poisson equation, $\nabla_{\perp}^{2} \varphi=$ $-\partial \rho / \partial t$, and using Eq.(7) we obtain an elliptic equation for the polarization field $\varphi$

$$
\nabla_{\perp}^{2} \varphi=\mathbf{V}_{E} \cdot \nabla \rho+\nabla_{\|} J_{\|}
$$

Here $\rho$ and $J_{\|}$are obtained from the marker distributions; these quantities are deposited on the computational grid at each time step. Based on representation (5), the gyrokinetic Poisson equation takes the form of

$$
\nabla_{\perp}^{2} \Phi-\left(1+\frac{1}{\tau}\right) \Phi=\int_{-\infty}^{+\infty}\left(h_{e}-h_{i}\right) d v_{\|}+Q(\Phi)
$$

where $Q(\Phi) \equiv \exp (\Phi)-\exp (-\Phi / \tau)-(1+1 / \tau) \Phi$ and $\tau=T_{i} / T_{e}$. The nonadiabatic response $h_{j}$ is normalized according to $c_{s} h_{j} / n_{0} \mapsto h_{j}$. The markers are evolved in phase space using the equations of motion (in gyrokinetic units)

$$
\frac{d \mathbf{r}}{d t}=v_{\|} \widehat{\mathbf{b}}_{0}+\widehat{\mathbf{b}}_{0} \times \nabla \Phi
$$

and

$$
\frac{d v_{\|}}{d t}=-Z_{j} \frac{m_{i}}{m_{j}} \nabla_{\|} \Phi
$$

In summary, the model equations for the conventional $\delta f$ scheme are the weight equation, Eq.(3), the gyrokinetic Poisson equation, Eq.(4), and the equations of motion, Eqs $(10,11)$. The corresponding equations for our kinetic electron scheme are the evolution equation for the marker weight associated with the nonadiabatic part of the electron distribution, Eq.(6), the elliptic equations $(9,8)$ for $\Phi$ and $\varphi=\partial \Phi / \partial t$, respectively; and the usual equations of motion, Eqs. $(10,11)$. The linear and nonlinear properties of these schemes are compared and discussed in the next section. 


\section{Numerical Results}

The linear dispersion relation based on the splitting scheme can be obtained as follows (intermediate results are presented in physical units rather than normalized units). Assuming perturbations of the form $\exp (i \mathbf{k} \cdot \mathbf{r}-i \omega t)$ and linearizing, the nonadiabatic response reads

$$
h_{j}=\left[\omega_{\star} g_{j}\left(\bar{v}_{\|}\right)+\sigma_{j} \omega\right] \frac{F_{M j}}{\omega-k_{\|} v_{\|}} \widetilde{\Phi},
$$

where $g_{j}\left(\bar{v}_{\|}\right)=1-\eta_{j}\left(1-\bar{v}_{\|}^{2}\right) / 2, \bar{v}_{\|}=v_{\|} / V_{t h j}, \omega_{\star}=\left(k_{y} \rho_{\mathrm{s}}\right) c_{s} / L_{n}$ is the drift frequency, $\sigma_{j}=Z_{j} T_{e} / T_{j}, \widetilde{\Phi}=e \Phi / T_{e}, \eta_{j}=L_{n} / L_{T_{j}}, L_{n}=\left(-d \ln n_{0} / d x\right)^{-1}$ and $L_{T_{j}}=\left(-d \ln T_{j} / d x\right)^{-1}$. Multiplying Eq.(12) by $v_{\|}^{k}$ and integrating over velocity space, one gets

$$
\int_{-\infty}^{+\infty} h_{j} v_{\|}^{k} d v_{\|}=-n_{0} \frac{\left(\sqrt{2} V_{t h j}\right)^{k}}{\omega_{\| j}}\left\{\left[\sigma_{j} \omega+\omega_{\star}\left(1-\eta_{j} / 2\right)\right] Z^{(k)}\left(\zeta_{j}\right)+\omega_{\star} \eta_{j} Z^{(k+2)}\left(\zeta_{j}\right)\right\} \widetilde{\Phi},
$$

where $\omega_{\| j} \equiv \sqrt{2} k_{\|} V_{t h j}, \zeta_{j} \equiv \omega / \omega_{\| j}$; we have defined

$$
Z^{(k)}(\zeta) \equiv \frac{1}{\sqrt{\pi}} \int_{-\infty}^{+\infty} \frac{x^{k}}{x-\zeta} \exp \left(-x^{2}\right) d x
$$

for nonnegative integer $k ; Z^{(0)}(\zeta) \equiv Z(\zeta)$ is the usual plasma dispersion function of Fried and Conte [7]. The particle density for species $j$ is

$$
\begin{aligned}
n_{j} & =n_{0} \exp \left(-\frac{q_{j}}{T_{j}} \Phi\right)+\int_{-\infty}^{+\infty} h_{j} d v_{\|}, \\
& =n_{0}\left(1-\sigma_{j} \widetilde{\Phi}\right)+\int_{-\infty}^{+\infty} h_{j} d v_{\|} .
\end{aligned}
$$

Substituting Eq.(15) in the gyrokinetic Poisson equation, $k_{y}{ }^{2} \rho_{\mathrm{s}}{ }^{2} \widetilde{\Phi}=\left(n_{i}-n_{e}\right) / n_{0}$, and using Eq.(13), we obtain the dispersion relation for electrostatic drift waves

$$
\left(1+\frac{1}{\tau}+b\right) \omega=-\omega\left[\zeta_{e} Z\left(\zeta_{e}\right)+\frac{1}{\tau} \zeta_{i} Z\left(\zeta_{i}\right)\right]+\omega_{\star}\left[\zeta_{e} R\left(\zeta_{e}\right)-\zeta_{i} R\left(\zeta_{i}\right)\right],
$$

where $R\left(\zeta_{j}\right) \equiv\left(1-\eta_{j} / 2\right) Z\left(\zeta_{j}\right)+\eta_{j} \zeta_{j}\left[1+\zeta_{j} Z\left(\zeta_{j}\right)\right] / 2$ and $b=k_{y}{ }^{2} \rho_{\mathrm{s}}{ }^{2}$. For benchmark purposes, the dispersion relation (16) has been solved numerically using Muller's algorithm [10] in the complex $\omega_{r}-\gamma$ plane. One consequence of the splitting scheme is that the original (linear) gyrokinetic Poisson equation, Eq.(4), is transformed into a nonlinear elliptic problem, Eq.(9), through the term $Q(\Phi)$. This nonlinear equation has been solved using a multigrid algorithm [8, 9]. Newton's method is used to treat the term $Q(\Phi)$ in Eq.(9); typically 2 to 3 Newton iterations are sufficient for convergence. For all the simulations presented in this paper, the multigrid relaxations have been carried out with a set of $6 V$ cycles, with $\nu=4$ relaxations on each grid level.

The equations of motion and the weight equation have been integrated using a fourth-order Runge-Kutta scheme. A linear interpolation has been used to deposit marker quantities (number density and current density) on the computational grid. 
We first discuss some linear simulation results. Figure 1 shows the linear growth rate obtained using the $\delta f$ scheme (triangles) and the splitting scheme (squares). The plain line is the numerical solution of the dispersion relation (16); the parameters are: $N_{i}=6765$ (number of ion markers); $N_{e}=6765$; system length $L=8$ for a grid with 64 nodes; the time step is $\Delta t=1.0$. The magnetic field tilt is $\theta=0.01$, and the electron and ion temperature-gradient parameters are $\eta_{e}=\eta_{i}=0$. The driving parameter, $\kappa=\rho_{s} / L_{n}$, has been varied between 0.05 and 0.13 . Although the splitting scheme results agree very well with the exact solution, the $\delta f$ scheme gives inaccurate results both for low and strong drives.

Figure 2 shows the power spectrum of the electrostatic potential for a fully nonlinear simulation, based on a time history of 5000 time steps, for the conventional $\delta f$ scheme (in Figs. $(2,3)$, $[\Phi]$ stands for $\left.|\Phi|^{2}\right)$. The spectrum peaks at $\omega_{r} / \omega_{c i} \simeq 0.06$. We note the presence of spurious modes in the high-frequency tail of the power spectrum. Figure 3 shows the power spectrum of $\Phi$ for the splitting scheme, using the same physical parameters, the same initial conditions in phase space and the same number of time steps as in Figure 2. The most dramatic aspect of Figure 3 is that the high-frequency part of $P(\omega)$ is now free of spurious modes. We expect this clean result to impact the particle number and energy conservation properties of the simulated plasma. This is confirmed below.

Figure 4 shows the absolute value of the time-integrated averaged weight of the electrons, defined as

$$
\left\langle\left\langle W_{e}\right\rangle\right\rangle \equiv \frac{1}{T} \int_{0}^{T} \frac{1}{N_{e}} \sum_{k=1}^{N_{e}} W_{k}(t) d t
$$

where $W_{k}(t)$ is the marker weight of the $k^{\text {th }}$ at time $t$, as a function of the drive, $\kappa$; here $T$ is the integration time which is chosen so that $\gamma_{\ell} T \gg 1$, where $\gamma_{\ell}$ is the linear growth rate of the fastest growing mode; in Figures 4 and 5, $T=2000$ and other parameters are the same as in Figure 3. For an infinite number of markers and an infinitesimally small time step one would get $\left\langle\left\langle W_{e}\right\rangle\right\rangle=0$. However, finite-size effects ('granularity' in temporal, configuration and velocity spaces) prevent perfect particle number conservation. As expected, the stronger the instability, the poorer the particle number conservation. However, since the parallel motion has been removed from the weight equation [Eq.(6)], the splitting scheme has a discrepancy in the number conservation two orders magnitude smaller that the conventional $\delta f$ scheme.

Figure 5 shows the time-integrated total energy, defined as

$$
\langle E\rangle \equiv \frac{1}{T} \int_{0}^{T}\left\{\frac{1}{2 L} \int_{0}^{L}\left|\nabla_{\perp} \Phi\right|^{2} d x+\frac{1}{2} \int_{-\infty}^{+\infty} v_{\|}^{2}\left(F_{i}+\frac{m_{e}}{m_{i}} F_{e}\right) d v_{\|}\right\} d t
$$

as a function of the driving parameter, $\kappa$. The splitting scheme achieves very good energy conservation, even when the drive is strong. The poor particle number and energy conservation properties of the $\delta f$ scheme can be intuitively understood in view of the high-frequency random kicks (Figure 2) experienced by the markers. Conversely, the clean power spectrum for the splitting scheme (Figure 3) translates in improved conservation properties. Although there are various ways to 'split' the electron distribution, the splitting scheme based on Eq.(5) yields an evolution equation for the marker weight which does not involve the free streaming contribution. Alternatively, writing the electron distribution function as $F_{e}=\left(e \Phi / T_{e}\right) F_{M e}+h_{e}$ yields a marker evolution equation which does involve a free streaming term with its associated noise and detrimental impact on the energy conservation. 


\section{Concluding Remarks}

The accurate treatment of electron dynamics in particle-in-cell simulations represents a major challenge since the fast electrons, $V_{t h e} / V_{t h i} \sim \sqrt{m_{i} / m_{e}} \gg 1$, imply a stringent condition on the time step. However, for low-frequency modes $[2,3]$ that are relevant to the anomalous transport in tokamaks [1] and stellarators [4], the bulk of the electrons do not "see" the waves; based on this observation, we have developed the splitting scheme, which segregates electrons into two subgroups (adiabatic electrons and nonadiabatic electrons). It has been shown that the splitting scheme has better linear and nonlinear properties than those of the conventional $\delta f$ scheme.

\section{Acknowledgments}

This research was supported by Contract No DE-AC02-76CH03073 and the Scientific Discovery through Advanced Computing (SciDAC) initiative (U.S. Department of Energy). 


\section{References}

[1] P. C. Liewer, Nucl. Fusion 25, 543 (1985).

[2] W. Horton, Handbook of Plasma Physics (Vol.2) (Elsevier Science, Amsterdam, 1984).

[3] W. Horton, Rev. Mod. Phys. 71, 735 (1999).

[4] F. Wagner and U. Stroth, Plasma Phys. Contr. Fusion, 35, 1321 (1993).

[5] R.E. Denton and M. Kotschenreuther, J. Comp. Phys. 119, 283 (1995).

[6] A. Brizard, J. Plasma Phys. 41, 541 (1989).

[7] B.D. Fried and S.D. Conte, Plasma Dispersion Function (Academic Press, New York, 1961).

[8] F. De la Vallee Poussin, SIAM J. of Num. Analysis, 5(2), 340 (1968).

[9] A. Settari and K. Aziz, SIAM J. of Num. Analysis, 10(3), 506 (1973).

[10] W.S Press, S.A. Teukolsky, W.T. Vetterling and B.P. Flannery, Numerical Recipes in Fortran (Cambridge University Press, New York, 1992). 
Figure 1 Linear growth rate for the standard $\delta f$ scheme (triangles) and for the splitting scheme (squares) as a function of $\kappa=\rho_{\mathrm{s}} / L_{n}$. The plain line is the numerical solution of the linear dispersion relation. The parameters are: $N_{e}=N_{i}=6765$, on a grid of length $L=8$ with 64 grid points; $\eta_{e}=\eta_{i}=0$ and $\theta=0.01$. Only the $N=1$ mode $\left(k_{\perp} \rho_{s} \simeq 0.78\right)$ is retained in the simulation. The initial configuration in phase space for the splitting scheme run and the $\delta f$ run are identical.

Figure 2 Power spectrum of the electrostatic potential based on a nonlinear simulation with 5000 time steps for the $\delta f$ scheme ; other parameters are $N_{i}=N_{e}=10946, \kappa=0.1$, $\theta=0.01,64$-grid of length $L=8$.

Figure 3 Same as Figure 2 but for the splitting scheme. The initial conditions in phase space and the physical parameters are identical to those of Figure 2.

Figure 4 Absolute value of the time-integrated average weight as a function of the drive, $\kappa$, for the standard $\delta f$ scheme (squares) and the splitting scheme (triangles); other parameters are the same as in Fig. 2 .

Figure 5 Time-integrated total energy as a function of the drive, $\kappa$, for the standard $\delta f$ scheme (squares) and the splitting scheme (triangles); other parameters are the same as in Fig.2. 


\section{FIG.1 Lewandowski}

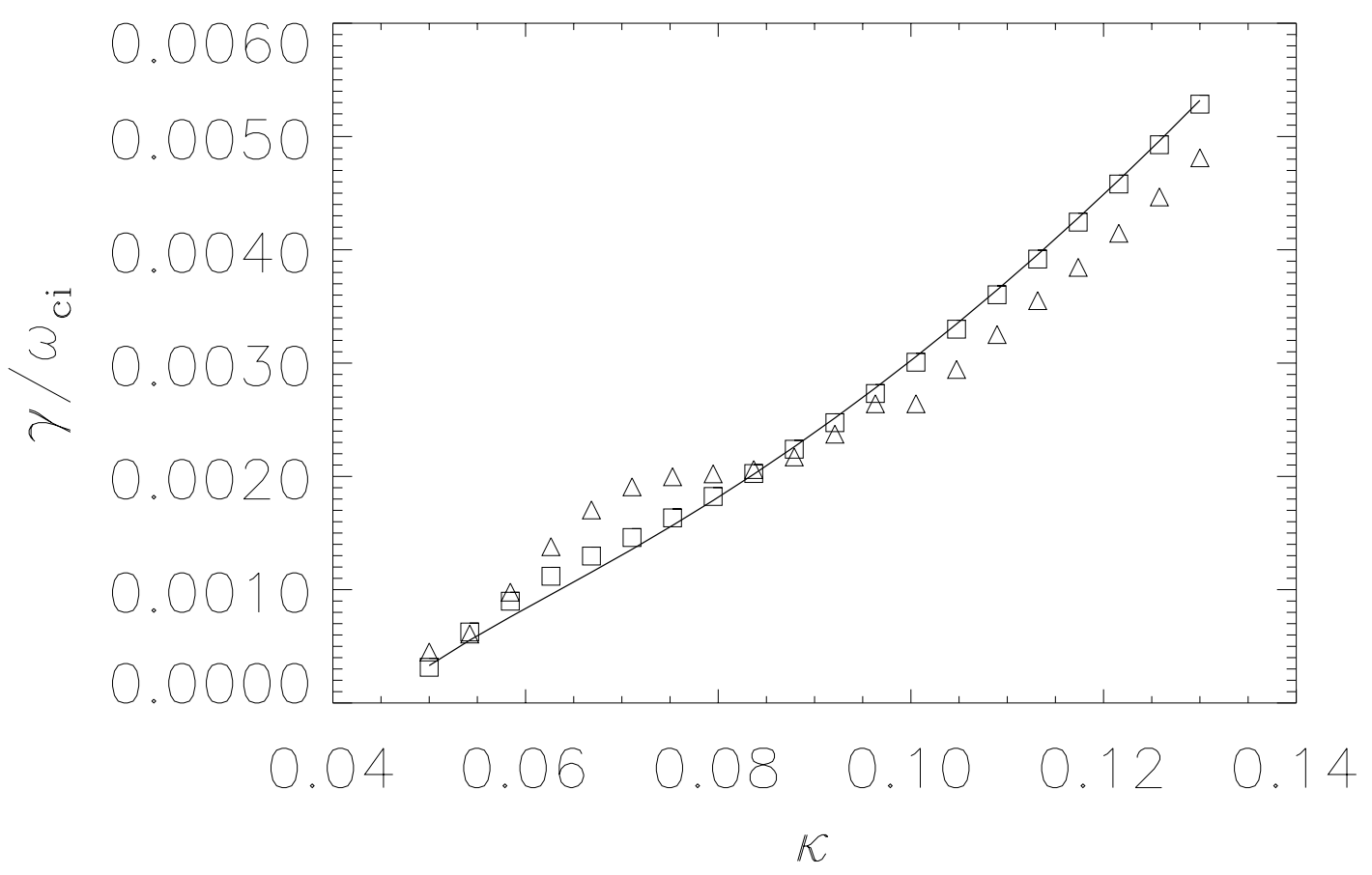




\section{FIG.2 Lewandowski}

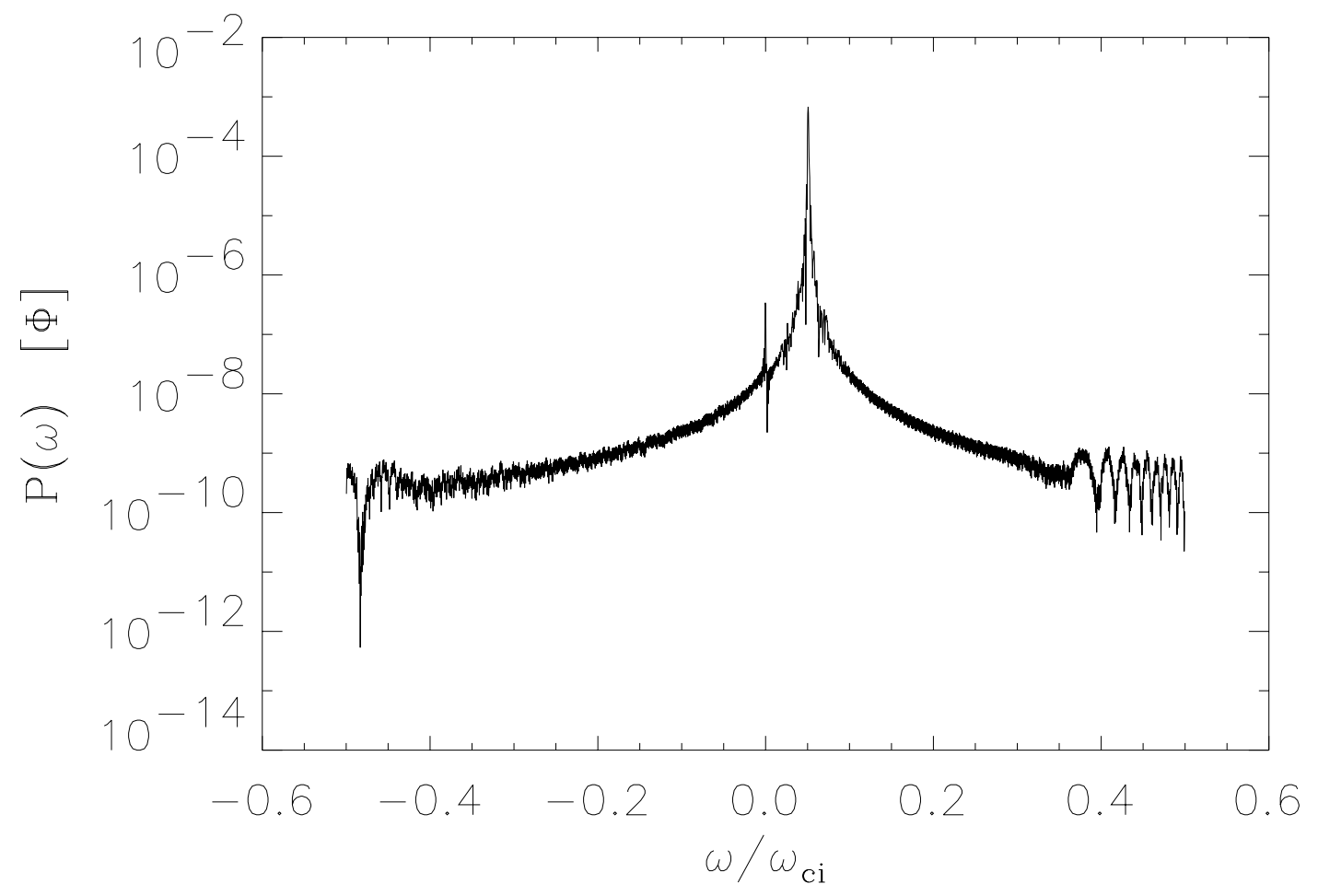




\section{FIG.3 Lewandowski}

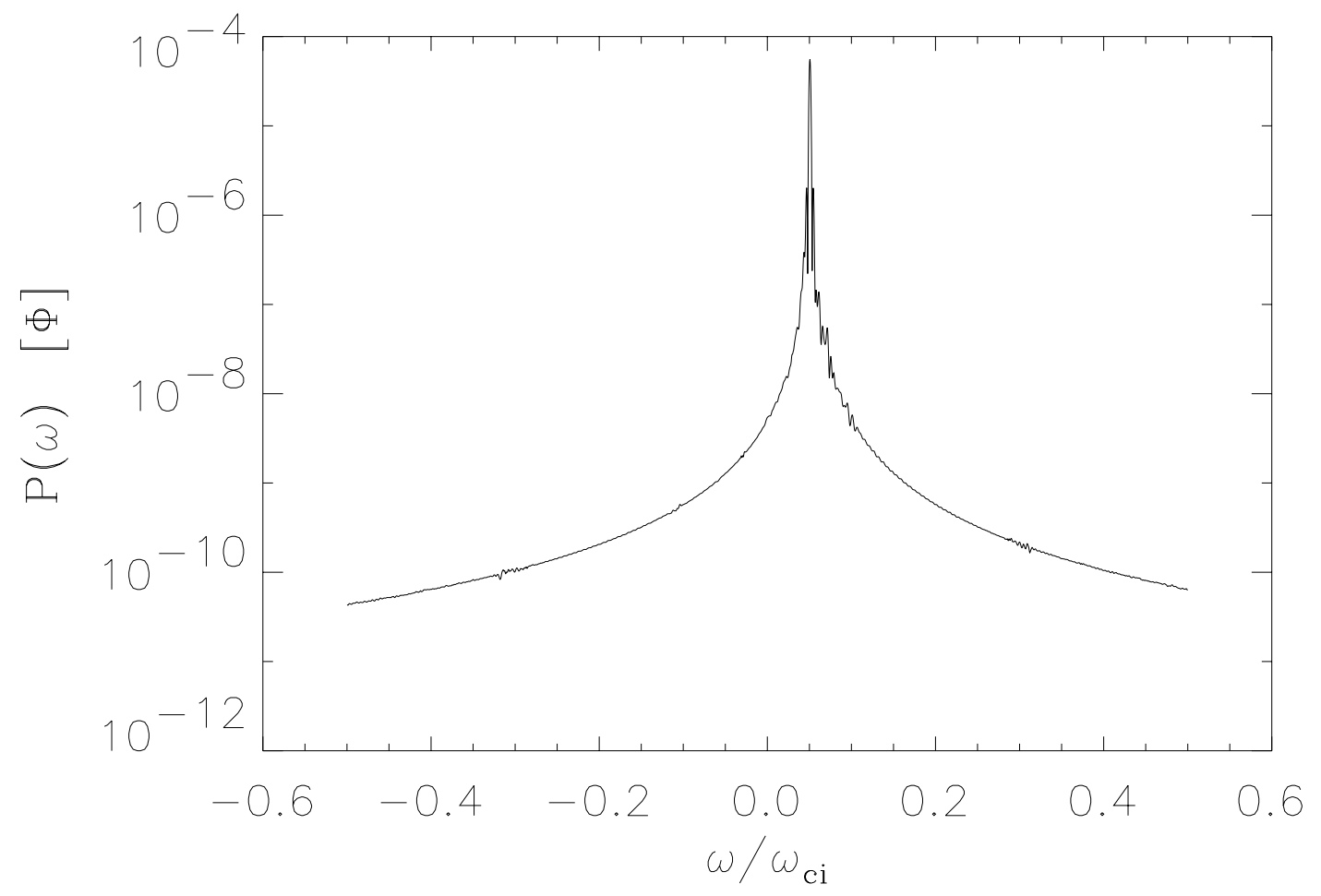




\section{FIG.4 Lewandowski}

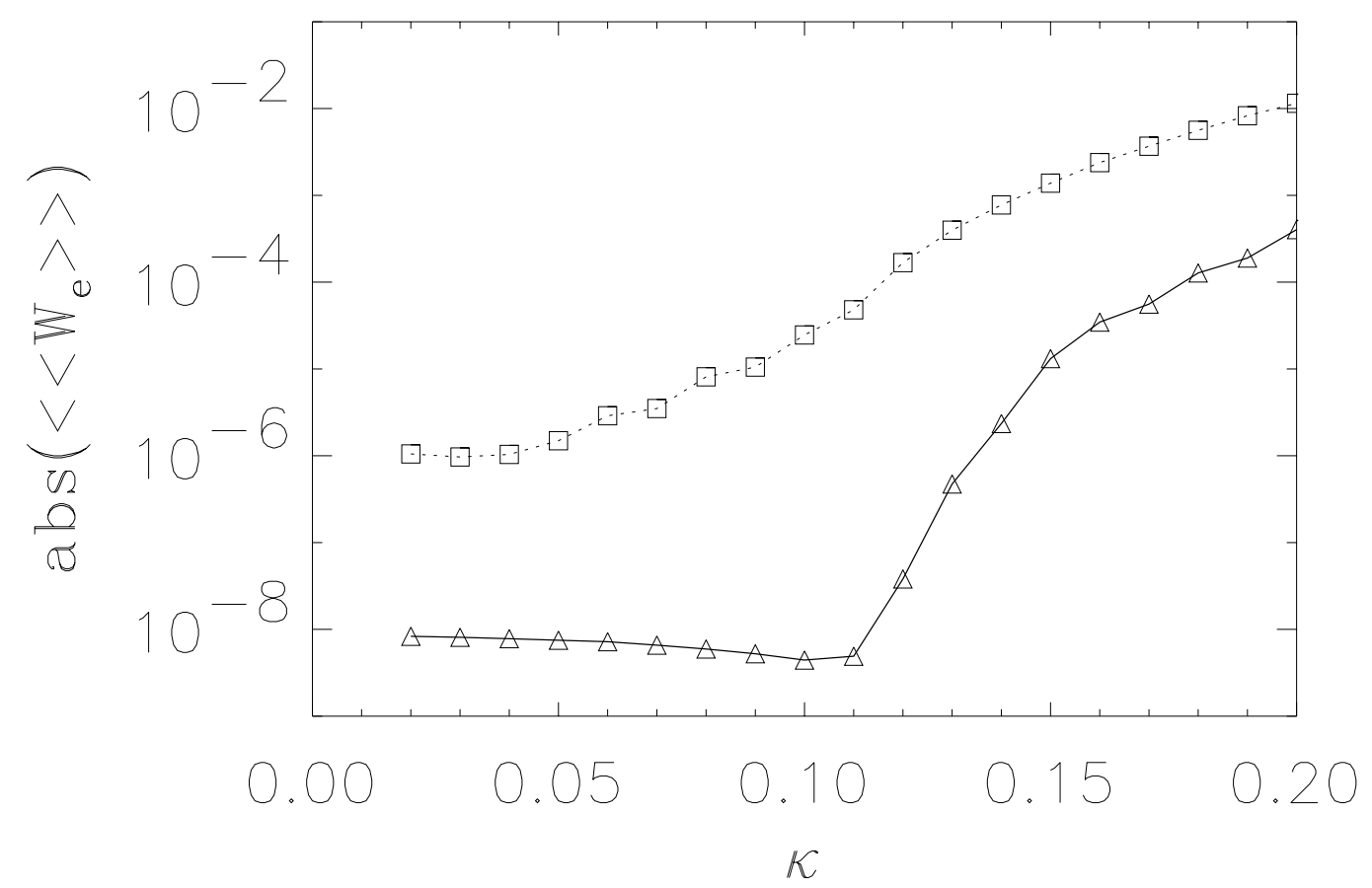




\section{FIG.5 Lewandowski}

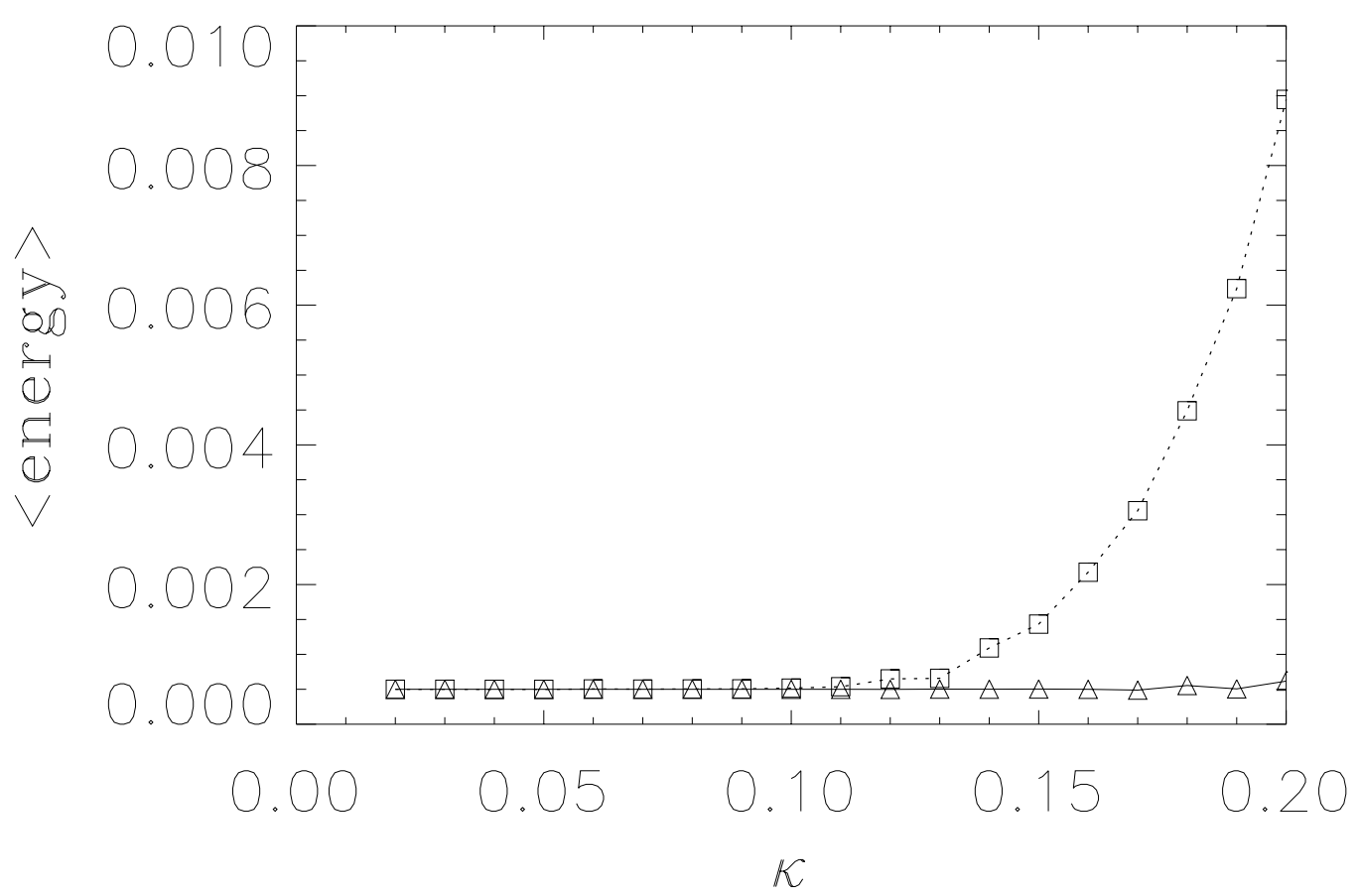




\section{External Distribution}

Plasma Research Laboratory, Australian National University, Australia

Professor I.R. Jones, Flinders University, Australia

Professor João Canalle, Instituto de Fisica DEQ/IF - UERJ, Brazil

Mr. Gerson O. Ludwig, Instituto Nacional de Pesquisas, Brazil

Dr. P.H. Sakanaka, Instituto Fisica, Brazil

The Librarian, Culham Laboratory, England

Mrs. S.A. Hutchinson, JET Library, England

Professor M.N. Bussac, Ecole Polytechnique, France

Librarian, Max-Planck-Institut für Plasmaphysik, Germany

Jolan Moldvai, Reports Library, MTA KFKI-ATKI, Hungary

Dr. P. Kaw, Institute for Plasma Research, India

Ms. P.J. Pathak, Librarian, Insitute for Plasma Research, India

Ms. Clelia De Palo, Associazione EURATOM-ENEA, Italy

Dr. G. Grosso, Instituto di Fisica del Plasma, Italy

Librarian, Naka Fusion Research Establishment, JAERI, Japan

Library, Plasma Physics Laboratory, Kyoto University, Japan

Research Information Center, National Institute for Fusion Science, Japan

Dr. O. Mitarai, Kyushu Tokai University, Japan

Dr. Jiangang Li, Institute of Plasma Physics, Chinese Academy of Sciences, People's Republic of China

Professor Yuping Huo, School of Physical Science and Technology, People's Republic of China

Library, Academia Sinica, Institute of Plasma Physics, People's Republic of China

Librarian, Institute of Physics, Chinese Academy of Sciences, People's Republic of China

Dr. S. Mirnov, TRINITI, Troitsk, Russian Federation, Russia

Dr. V.S. Strelkov, Kurchatov Institute, Russian Federation, Russia

Professor Peter Lukac, Katedra Fyziky Plazmy MFF UK, Mlynska dolina F-2, Komenskeho Univerzita, SK-842 15 Bratislava, Slovakia

Dr. G.S. Lee, Korea Basic Science Institute, South Korea

Institute for Plasma Research, University of Maryland, USA

Librarian, Fusion Energy Division, Oak Ridge National Laboratory, USA

Librarian, Institute of Fusion Studies, University of Texas, USA

Librarian, Magnetic Fusion Program, Lawrence Livermore National Laboratory, USA

Library, General Atomics, USA

Plasma Physics Group, Fusion Energy Research Program, University of California at San Diego, USA

Plasma Physics Library, Columbia University, USA

Alkesh Punjabi, Center for Fusion Research and Training, Hampton University, USA

Dr. W.M. Stacey, Fusion Research Center, Georgia Institute of Technology, USA

Dr. John Willis, U.S. Department of Energy, Office of Fusion Energy Sciences, USA

Mr. Paul H. Wright, Indianapolis, Indiana, USA 
The Princeton Plasma Physics Laboratory is operated by Princeton University under contract with the U.S. Department of Energy.

\author{
Information Services \\ Princeton Plasma Physics Laboratory \\ P.O. Box 451 \\ Princeton, NJ 08543
}

Phone: 609-243-2750

Fax: 609-243-2751

e-mail: pppl_info@pppl.gov

Internet Address: http://www.pppl.gov 